\title{
On extended graphical calculus for categorified quantum $\operatorname{sl}(n)$
}

\author{
Marko Stošić
}

\begin{abstract}
We study the properties of the extended graphical calculus for categorified quantum $\operatorname{sl}(n)$. The main results include proofs of Reidemeister 2 and Reidemeister 3-like moves involving strands corresponding to arbitrary thicknesses and arbitrary colors - the results that were anounced in [12.
\end{abstract}

Keywords: categorification; quantum groups; diagrammatic calculus

\section{Introduction}

In recent years there has been a lot of work on diagrammatic categorification of quantum groups, initiated by Lauda's diagrammatic categorification [7] (see also [3]) of Lusztig's idempotented version of $\dot{U}_{q}\left(\mathfrak{s l}_{2}\right)$. This was extended by Khovanov and Lauda in [5] to $\dot{U}_{q}\left(\mathfrak{s l}_{n}\right)$ and also in [4] to the positive half of an arbitrary quantum group $U_{q}^{+}(\mathfrak{g})$.

The general framework of these constructions is to define a certain 2category $\mathcal{U}$ whose 1 -morphisms categorify generators of a quantum group, and whose 2-morphisms are $\mathbb{K}$-linear combinations of certain planar diagrams modulo local relations, with $\mathbb{K}$ being a field. Then a 2-category $\dot{\mathcal{U}}$ is defined as the Karoubi envelope of the 2-category $\mathcal{U}$, i.e. the smallest category containing $\mathcal{U}$ in which all idempotent 2-morphisms split. Finally, it is shown that the split Grothendieck group of $\dot{\mathcal{U}}$ is isomorphic to the corresponding quantum group.

For the categorification of $U_{q}^{+}(\mathfrak{g})$, the 2-categories $\mathcal{U}$ and $\dot{\mathcal{U}}$ have a single object. Thus one can see them as monoidal 1-categories. Since in this paper we are interested in categorifications of positive halves of quantum groups, we shall always assume that $\mathcal{U}$ and $\dot{\mathcal{U}}$ are monoidal (1-)categories.

The extension of the diagrammatic calculus - so-called thick calculus was introduced in [6] in the case of quantum $\mathfrak{s l}_{2}$. With thick calculus one can work directly in $\dot{\mathcal{U}}$, and not just in $\mathcal{U}$. The thick calculus can be extended directly to the case of quantum $\mathfrak{s l}_{n}$ (see [12]). 
The consequence of [6], and of the thick calculus, is that now one can take $\mathbb{Z}$-linear combinations of planar diagrams as morphisms of $\mathcal{U}$, because the idempotents being added have no denominators. In this paper, we study the properties of the thick calculus of the category $\dot{\mathcal{U}}_{n}^{+}$that categorifies the positive half of the quantum $\mathfrak{s l}_{n}$. In particular, the main advantage of this approach is that the category $\dot{\mathcal{U}}_{n}^{+}$is defined over the ring of integers.

Thick calculus already had many applications. This includes the computation of the indecomposable objects of $\dot{\mathcal{U}}_{3}^{+}$, and consequently the categorification of the Lusztig canonical basis for the positive half of $\dot{U}_{q}\left(\mathfrak{s l}_{3}\right)$ [12]. Previous results on this topic were obtained when the category is defined over a field, i.e. when 1-morphisms are $\mathbb{K}$-linear combinations of planar diagrams, for some characteristic zero field $\mathbb{K}$ (see [1, 3, 13]). Furthermore, thick calculus was also used in the combinatorial categorification of $\mathfrak{s l}_{n}$ link invariants of Queffelec and Rose [11], via categorical skew-Howe duality.

In this paper some of the new relations in the thick calculus of $\mathfrak{s l}_{n}$ are proved. This mainly includes the proofs or Reidemeister 2 and Reidemeister 3 - like moves for strands involving arbitrary thicknesses and labels, the results that were anounced in [12. Moreover, we also prove some additional relations for passing of a strand through another thick strand labeled by adjacent colors.

The results are combinatorial and rely heavily on properties of Schur polynomials, as well as on the numerous properties of the thick diagrammatical calculus both for quantum $\mathfrak{s l}_{2}$ [6] and quantum $\mathfrak{s l}_{n}$ from [12]. In order to make the paper as self-contained as possible, in the first part we recall the basic definitions of the category $\dot{\mathcal{U}}_{n}^{+}$and of the thick calculus, together with some basic properties that will be used in the proofs. Mainly we follow [12. Sections 4 and 5 contain the proofs of the Reidemeister 2 and Reimdeister 3 - like moves, respectively, while in Section [6 we prove some additional thick calculus relations as consequences of our main result.

\section{Acknowledgments}

I am thankful to Mikhail Khovanov on his comments and suggestions on the early version of this paper. This work was partially supported by the Ministry of Education and Science of Serbia, project no. 174012, and also by the ERC Starting Grant no. 335739 "Quantum fields and knot homologies" funded by the European Research Council under the European Unions Seventh Framework Programme. The final part of the work on this project was done while visiting Institute des Hautes Etudes Scientifiques (IHES), at Bures-sur-Yvette, France, and Max-Planck Institute for Mathematics in Bonn, Germany, and I would like to thank both institutions for hospitality and excellent working atmosphere. 


\section{$2 U_{q}^{+}\left(\mathfrak{s l}_{n}\right)$}

In this section we define the positive half of quantum $\mathfrak{s l}_{n}-\operatorname{denoted} U_{q}^{+}\left(\mathfrak{s l}_{n}\right)$. We also give some of its combinatorial properties in the case $n=3$.

Let $n \geq 2$ be fixed. The index set of quantum $\mathfrak{s l}_{n}$ is $I=\{1,2, \ldots, n-1\}$. An inner product is defined on $\mathbb{Z}[I]$ by setting

$$
i \cdot j=\left\{\begin{aligned}
2, & i=j \\
-1, & |i-j|=1 \\
0, & |i-j| \geq 2
\end{aligned}\right.
$$

for $i, j \in I$.

$U_{q}^{+}\left(\mathfrak{s l}_{n}\right)$ is a $\mathbb{Q}(q)$-algebra generated by $E_{1}, E_{2}, \ldots, E_{n-1}$ modulo relations:

$$
\begin{aligned}
E_{i}^{2} E_{j}+E_{j} E_{i}^{2} & =[2] E_{i} E_{j} E_{i}, \quad i \cdot j=-1, \\
E_{i} E_{j} & =E_{j} E_{i}, \quad i \cdot j=0 .
\end{aligned}
$$

The quantum integers and binomial coefficients are given by:

$$
\begin{aligned}
{[n] } & =\frac{q^{n}-q^{-n}}{q-q^{-1}}, \\
{[n] ! } & =[n][n-1] \cdots[2][1], \\
{\left[\begin{array}{l}
n \\
k
\end{array}\right] } & =\frac{[n] !}{[k] ![n-k] !} .
\end{aligned}
$$

The divided powers of the generators are defined by

$$
E_{i}^{(a)}:=\frac{E_{i}^{a}}{[a] !}, \quad a \geq 0, \quad i=1, \ldots, n-1 .
$$

The divided powers satisfy:

$$
\begin{aligned}
E_{i}^{(a)} E_{j}^{(b)} & =E_{j}^{(b)} E_{i}^{(a)}, \quad i \cdot j=0, \\
E_{i}^{(a)} E_{i}^{(b)} & =\left[\begin{array}{c}
a+b \\
a
\end{array}\right] E_{i}^{(a+b)},
\end{aligned}
$$

and the quantum Serre relations

$$
E_{i}^{(2)} E_{j}+E_{j} E_{i}^{(2)}=E_{i} E_{j} E_{i}, \quad i \cdot j=-1 .
$$

The integral form ${ }_{\mathbb{Z}} U_{q}^{+}\left(\mathfrak{s l}_{n}\right)$ is the $\mathbb{Z}\left[q, q^{-1}\right]$-subalgebra of $U_{q}^{+}\left(\mathfrak{s l}_{n}\right)$ generated by $E_{i}^{(a)}$, for all $i=1, \ldots, n-1$ and $a \geq 0$. 


\section{The category $\dot{\mathcal{U}}_{n}^{+}$}

A categorification of the positive half of quantum $\mathfrak{s l}_{n}$ (and also of an arbitrary quantum group $U_{q}^{+}(\mathfrak{g})$ ) was defined in [4, though in this paper we prefer the description found in [5] in terms of a diagrammatic category $\mathcal{U}_{n}^{+}$. Before going to the definition of $\mathcal{U}_{n}^{+}$, first we recall some notation and explain the diagrams that appear in its definition (see also [5]).

Let $n \geq 2$ be fixed. We refer to the elements of the set $\{1, \ldots, n-1\}$ as colors. Let Seq denote the set of all finite sequence of colors. For $\nu=\left(\nu_{1}, \ldots, \nu_{n-1}\right) \in \mathbb{N}^{n-1}$ let $\operatorname{Seq}(\nu)$ denote the set of all sequences $\underline{i}=$ $\left(i_{1}, \ldots, i_{k}\right) \in$ Seq such that $\sharp\left\{j \mid i_{j}=l\right\}=\nu_{l}$, for all $l=1, \ldots, n-1$. Note that $k=\sum_{l} \nu_{l}$.

We will use the following notion of planar diagrams: We consider collections of arcs on the plane connecting the points $\{1,2, \ldots, k\} \subset \mathbb{R}$ in one horizontal line to the points $\{1,2, \ldots, k\} \subset \mathbb{R}$ in another horizontal line. Each arc is labelled by a number from the set $\{1, \ldots, n-1\}$ (called the color of the arc). We require that arcs have no critical points when projected to $y$-axis. Arcs can intersect, but no triple intersections are allowed. Finally, an arc can carry dots.

The following is an example of a planar diagram:

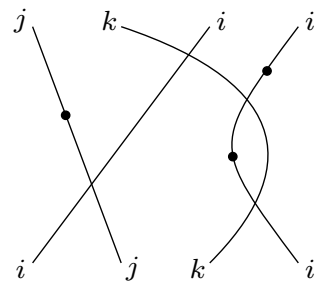

We identify two planar diagrams if there exists an isotopy between them that does not create critical points for the projection onto the $y$-axis.

Since we are not allowing the arcs to have critical points when projected to $y$-axis, we can assume that they are always oriented upwards. We think of a planar diagram as going from its bottom boundary (a sequence of colors) to its top boundary. We read the colors on each boundary from left to right.

Each diagram has a degree defined as follows. The degree of a dot is equal to 2. The degree of a crossing between two arcs that are colored $i$ and $j$ is equal to $-i \cdot j$. In other words, for $i=j$ the degree of a crossing is equal to -2 , for $|i-j|=1$ (adjacent colors) the degree of a crossing is equal to 1 , while for $|i-j| \geq 2$ (distant colors) the degree of a crossing is equal to 0 . Finally, the degree of a diagram is obtained by summing the contributions coming from all dots and all crossings. 


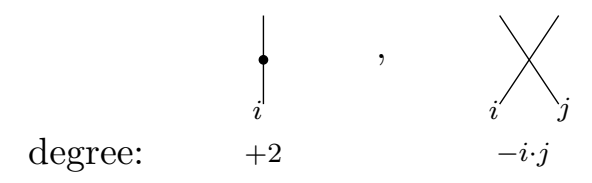

We also use the following shorthand for a collection of dots on a strand.

$$
\left.{ }_{d} \mid=\vdots \vdots\right\} d
$$

\subsection{The category $\mathcal{U}_{n}^{+}$}

$\mathcal{U}_{n}^{+}$is the monoidal $\mathbb{Z}$-linear additive category whose objects and morphisms are the following:

- objects: for each $\underline{i}=\left(i_{1}, \ldots, i_{k}\right) \in$ Seq and $t \in \mathbb{Z}$, we define $\mathcal{E}_{\underline{i}}\{t\}:=$ $\mathcal{E}_{i_{1}} \ldots \mathcal{E}_{i_{k}}\{t\}$. An object of $\mathcal{U}_{n}^{+}$is a formal finite direct sum of $\mathcal{E}_{\underline{i}}\{t\}$, with $i \in$ Seq and $t \in \mathbb{Z}$.

- morphisms: for $\underline{i}=\left(i_{1}, \ldots, i_{k}\right) \in \operatorname{Seq}(\nu)$ and $\underline{j}=\left(j_{1}, \ldots, j_{l}\right) \in \operatorname{Seq}(\mu)$ the set $\operatorname{Hom}\left(\mathcal{E}_{\underline{i}}\{t\}, \mathcal{E}_{\underline{j}}\left\{t^{\prime}\right\}\right)$ is empty, unless $\nu=\mu$. If $\nu=\mu$ (and consequently $k=l$ ), the morphisms from $\mathcal{E}_{\underline{i}}\{t\}$ to $\mathcal{E}_{j}\left\{t^{\prime}\right\}$ consist of finite $\mathbb{Z}$-linear combinations of planar diagrams going from $\underline{i}$ to $\underline{j}$, of degree $t-t^{\prime}$, modulo the following set of homogeneous local relations:

$$
\begin{aligned}
& X_{i}-X_{i}=X_{i}-Y_{i}=\left.\left.\right|_{i}\right|_{i} \\
& X_{i}=0, \quad X_{i}=X_{i}
\end{aligned}
$$

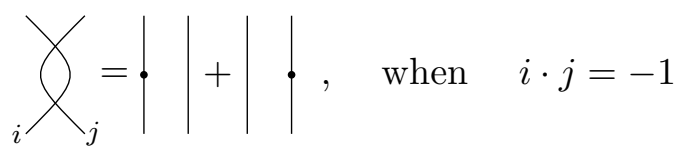

$$
\begin{aligned}
& X_{i}=\left.\left.\right|_{i}\right|_{j}, \text { when } i \cdot j=0
\end{aligned}
$$



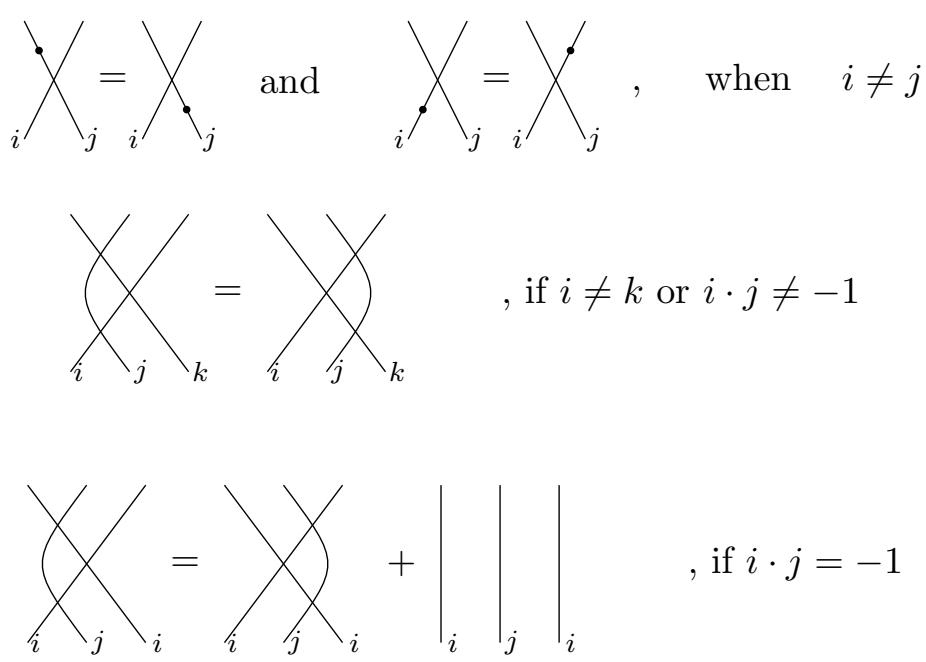

This ends the definition of $\mathcal{U}_{n}^{+}$.

As an example of a morphism, a diagram from (므) represents a morphism in $\operatorname{Hom}\left(\mathcal{E}_{i} \mathcal{E}_{j} \mathcal{E}_{k} \mathcal{E}_{i}\{t\}, \mathcal{E}_{j} \mathcal{E}_{k} \mathcal{E}_{i} \mathcal{E}_{i}\{t+2\}\right)$.

We have the following relation in $\mathcal{U}_{n}^{+}$:

Proposition 1 (Dot Migration) [7, Proposition 5.2] We have

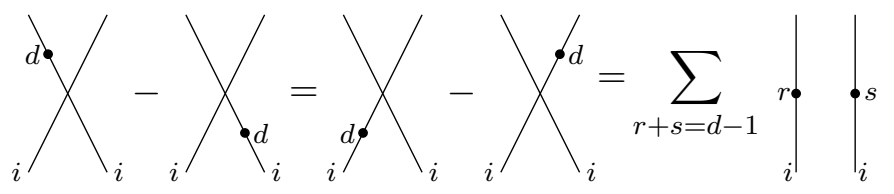

\subsection{The category $\dot{\mathcal{U}}_{n}^{+}$and thick calculus}

In [6], the extension of the calculus to thick edges have been introduced. Thick lines categorify the divided powers $E_{i}^{(a)}$ (see below and Section 4 of [6]).

For a category $\mathcal{C}$, the Karoubi envelope $\operatorname{Kar}(\mathcal{C})$ is the smallest category containing $\mathcal{C}$, such that all idempotents split (for more details, see e.g. Section 3.4 of [6]).

We define the category $\dot{\mathcal{U}}_{n}^{+}$as the Karoubi envelope of the category $\mathcal{U}_{n}^{+}$.

As in [6], the category $\dot{\mathcal{U}}_{n}^{+}$categorifies $U_{q}^{+}\left(\mathfrak{s l}_{n}\right)$, in a sense that its split Grothendieck group is isomorphic to the integral form of $U_{q}^{+}\left(\mathfrak{s l}_{n}\right)$. The isomorphism sends the class of $\mathcal{E}_{i}^{(a)}$ to the generator $E_{i}^{(a)}$ of $U_{q}^{+}\left(\mathfrak{s l}_{n}\right)$. 
In the category $\dot{\mathcal{U}}_{n}^{+}$, the planar diagrams with thick edges from above can be interpreted as morphisms whose bottom and top end correspond to certain objects of $\dot{\mathcal{U}}_{n}^{+}$. In particular, the object corresponding to bottom (or the top end) of an arc of color $i$ and thickness $a$ is denoted $\mathcal{E}_{i}^{(a)}$.

A thick line of color $i$ is defined as the identity morphism:

$$
\left.\right|_{a}: \mathcal{E}_{i}^{(a)} \longrightarrow \mathcal{E}_{i}^{(a)}
$$

It is given explicitly in terms of "ordinary" lines from above - see [6, Equation (2.18)], and drawn as a strand with an additional label (natural number) $a$ (also called the thickness of a strand). In particular, the ordinary strands from above correspond to the case $a=1$, and are also called thin edges or thin strands. We refer the reader to [6], in particular Sections 2 and 4 , for more details. Here we just recall the basic facts that will be used later on.

Trivalent vertices of a single color are now allowed in our planar diagrams, as long as the sum of thicknesses of the incoming edges is equal to the sum of thicknesses of the outgoing edges. These are called splitters in [6]. The trivalent vertices (for any color $i$ - the labels on the pictures below represent thicknesses)

$$
{ }_{a}^{a+b} \bigcup_{b}: \mathcal{E}_{i}^{(a)} \mathcal{E}_{i}^{(b)} \rightarrow \mathcal{E}_{i}^{(a+b)}{ }^{a} \bigvee_{a+b}^{b}: \mathcal{E}_{i}^{(a+b)} \rightarrow \mathcal{E}_{i}^{(a)} \mathcal{E}_{i}^{(b)}
$$

correspond to the projection and inclusion maps, respectively, obtained from the decomposition:

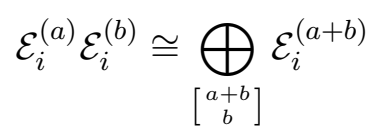

The degrees of both of this two vertices are equal to $-a b$, which explains which summands must be involved in these morphisms. The explicit definitions are given in [6, pp. 15].

These morphisms may be composed, and in particular they can be used to define the thick crossing:

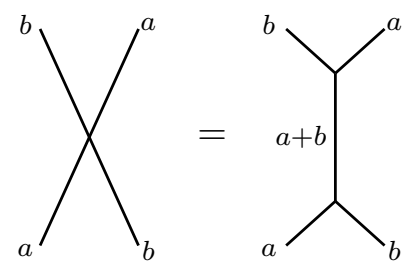




\subsubsection{Some properties of the thick calculus}

Below we give some of the basic properties of thick edges that we shall use in this paper (see [6] for more details). Note that the labels of the strands below denote thickness. All relations hold under horizontal and vertical flips, because of the symmetries on $\mathcal{U}$.

Proposition 2 (Associativity of splitters) [6, Proposition 2.2.4] For arbitrary color $i$ we have the following:

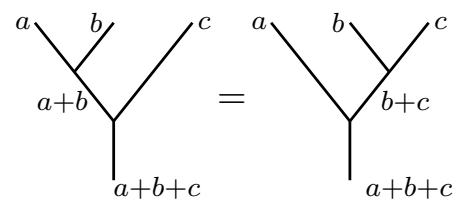

In particular, the above Associativity of Splitters imply that the multisplitters, i.e. splitting of a thick line into three or more strands, is welldefined.

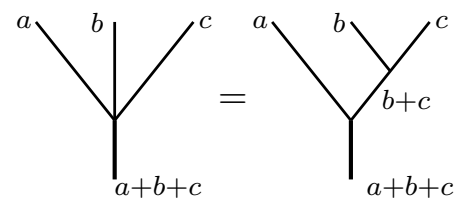

Proposition 3 (Pitchfork lemma) [6] For any two colors $i$ (drawn as a thick line) and $j$ (drawn dashed) we have:

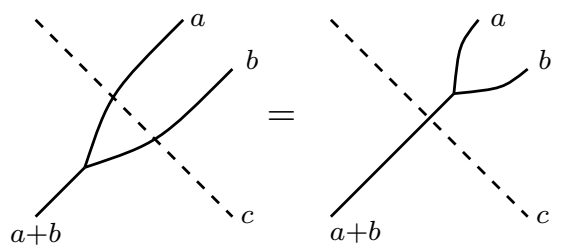

Proposition 4 (Opening of a Thick Edge) [6, Proposition 2.2.5] For any color $i$ we have:
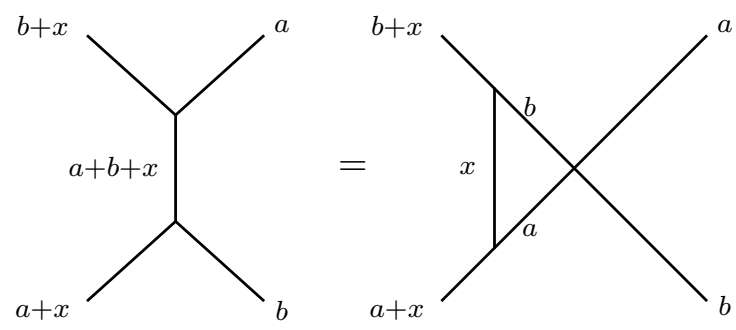


\subsection{Schur polynomials and decorations of thick lines}

Just as we can decorate thin strands with dots, we can decorate thick lines with symmetric polynomials. These correspond to symmetric polynomials in dots on thin edges involved in the definition of a thick line (for a precise definition, see [6]). For notational convenience we will only decorate thick strands with Schur polynomials, which form an additive basis of the ring of symmetric polynomials.

\subsubsection{Schur polynomials}

Here we recall briefly the definition and some basic notation and properties of Schur polynomials. For more details, see e.g. [2, 6, 10].

By a partition $\alpha=\left(\alpha_{1}, \ldots, \alpha_{k}\right)$, we mean a non-increasing sequence of non-negative integers. We identify two partitions if they differ by a sequence of zeros at the end. We set $|\alpha|=\sum_{i} \alpha_{i}$. If for some $a$ we have $\alpha_{a+1}=0$, we say that $\alpha$ has at most $a$ parts. We denote the set of all partitions with at most $a$ parts by $P(a)$. Furthermore, by $P(a, b)$ we denote the subset of all partitions $\alpha$ from $P(a)$ such that $\alpha_{1} \leq b$. In other words, $P(a, b)$ consists of partitions fitting inside a rectangle with $a$ rows and $b$ columns. The partition corresponding to this rectangle we denote by $K_{a, b}$, i.e. $K_{a, b}=(\underbrace{b, b, \ldots, b}_{a})$.

We shall need to express quantum binomial coefficients as a sum over partitions fitting inside a rectangle. For any two nonnegative integers $a$ and $b$ we have:

$$
\left[\begin{array}{c}
a+b \\
a
\end{array}\right]=\sum_{\alpha \in P(a, b)} q^{2|\alpha|-a b} .
$$

By $\bar{\alpha}$ we denote the dual (conjugate) partition of $\alpha$, i.e. $\alpha_{j}=\sharp\left\{i \mid \alpha_{i} \geq j\right\}$. If $\alpha \in P(a, b)$, we define partition $\hat{\alpha}$ by $\hat{\alpha}=\overline{\left(b-\alpha_{a}, \ldots, b-\alpha_{1}\right)}$. Note that if $\alpha \in P(a, b)$, then $\bar{\alpha} \in P(b, a)$ and $\hat{\alpha} \in P(b, a)$.

For any partition $\alpha \in P(a)$, the Schur polynomial $\pi_{\alpha}$ is given by the formula:

$$
\pi_{\alpha}\left(x_{1}, x_{2}, \ldots, x_{a}\right)=\frac{\left|x_{i}^{\alpha_{j}+a-j}\right|}{\Delta},
$$

where $\Delta=\prod_{1 \leq r \leq s \leq a}\left(x_{r}-x_{s}\right)$, and $\left|x_{i}^{\alpha_{j}+a-j}\right|$ is the determinant of the $a \times a$ matrix whose $(i, j)$ entry is $x_{i}^{\alpha_{j}+a-j}$. We extend our notation, so that $\pi_{\alpha}\left(x_{1}, x_{2}, \ldots, x_{a}\right)=0$ is some entry of $\alpha$ is negative ( $\alpha$ is not a partition then), or if $\alpha_{a+1}>0$.

For two partitions $\alpha$ and $\gamma$, we say that $\alpha \subset \gamma$ if $\alpha_{i} \leq \gamma_{i}$ for all $i \geq 1$.

For three partitions $\alpha, \beta$ and $\gamma$, the Littlewood-Richardson coefficients $c_{\alpha, \beta}^{\gamma}$ are given by:

$$
\pi_{\alpha} \pi_{\beta}=\sum_{\gamma} c_{\alpha, \beta}^{\gamma} \pi_{\gamma}
$$


The coefficients $c_{\alpha, \beta}^{\gamma}$ are nonnegative integers that can be nonzero only when $|\gamma|=|\alpha|+|\beta|$. Also, $c_{\alpha, \beta}^{\gamma} \neq 0$ only when $\alpha \subset \gamma$ and $\beta \subset \gamma$. In particular:

$\alpha \in P(a, x), \beta \in P(b, y)$ and $c_{\alpha, \beta}^{\gamma} \neq 0$, imply $\gamma \in P(a+b, x+y)$.

The Littlewood-Richardson coefficients can be naturally extended for more than three partitions: for partitions $\alpha_{1}, \ldots, \alpha_{k}$ and $\beta$, with $k \geq 2$, we define $c_{\alpha_{1}, \ldots, \alpha_{k}}^{\beta}$ by:

$$
\pi_{\alpha_{1}} \ldots \pi_{\alpha_{k}}=\sum_{\beta} c_{\alpha_{1}, \ldots, \alpha_{k}}^{\beta} \pi_{\beta} .
$$

For two partitions $\alpha$ and $\gamma$, we define skew-Schur polynomial $\pi_{\gamma / \alpha}$ by:

$$
\pi_{\gamma / \alpha}=\sum_{\beta} c_{\alpha, \beta}^{\gamma} \pi_{\beta}
$$

It can be nonzero only when $\alpha \subset \gamma$.

If $\gamma=\left(\gamma_{1}, \ldots, \gamma_{a}\right) \subset K_{a, b}$, then by $K_{a, b}-\gamma$ we denote the partition $\left(b-\gamma_{a}, \ldots, b-\gamma_{1}\right)$. For a partition $\nu \in P(a)$, by $\nu+K_{a, b}$ we denote the partition $\left(\nu_{1}+b, \ldots, \nu_{a}+b\right)$. Furthermore, for every two partitions $\psi \in P(a)$ and $\gamma \in P(a, b)$, we have that $c_{\gamma, \psi}^{\nu+K_{a, b}}=c_{\nu, K_{a, b}-\gamma}^{\psi}$. In particular, if $\nu=\emptyset$, one has $c_{\gamma, \psi}^{K_{a, b}}=c_{\emptyset, K_{a, b}-\gamma}^{\psi}=\delta_{\psi, K_{a, b}-\gamma}$, and so $\pi_{K_{a, b} / \gamma}=\pi_{K_{a, b}-\gamma}$.

The elementary symmetric polynomials $\varepsilon_{m}\left(x_{1}, \ldots, x_{a}\right)$, for $m=0, \ldots, a$ are special Schur polynomials: $\varepsilon_{m}\left(x_{1}, \ldots, x_{a}\right)=\pi_{(\underbrace{1,1, \ldots, 1}_{m}}\left(x_{1}, \ldots, x_{a}\right)$. For $m<0$ or $m>a$, we have $\varepsilon_{m}\left(x_{1}, \ldots, x_{a}\right)=0$.

The Schur polynomials can be conveniently expressed as a determinant of a matrix whose entries are the elementary symmetric polynomials, by the following Giambelli formula: for a partition $\alpha=\left(\alpha_{1}, \ldots, \alpha_{a}\right)$, we have

$$
\pi_{\bar{\alpha}}=\operatorname{det}\left[\varepsilon_{\alpha_{i}+j-i}\right]_{i, j=1}^{a} .
$$

\subsubsection{Decorated thick edges}

Here we recall some of the basic properties of decorated thick lines that we shall need in this paper. For more details see [6].

A thick line of thickness $a$ can be decorated with any Schur polynomial $\pi_{\alpha}$. For $\alpha \notin P(a)$, the resulting morphism is zero. For $\alpha \in P(a)$ of the form $\alpha=\left(\alpha_{1}, \alpha_{2}, \ldots, \alpha_{a}\right)$, one can express the decoration of a thick line in terms of thin lines and dots as follows

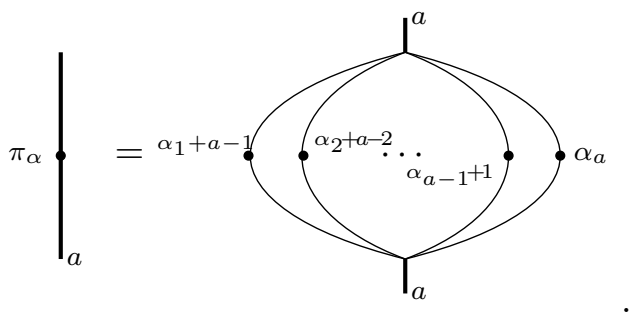


From the definition of the Littlewood-Richardson coefficient, we have

$$
\left.\begin{array}{c}
\pi_{\beta} \\
\pi_{\alpha}
\end{array}\right\}=\sum_{\gamma} c_{\alpha \beta}^{\gamma} \pi_{\gamma} \downarrow
$$

By "exploding" a thick edge into thin edges, we obtain diagrams that are antisymmetric with respect to the exchange of dots on two neighboring strands:

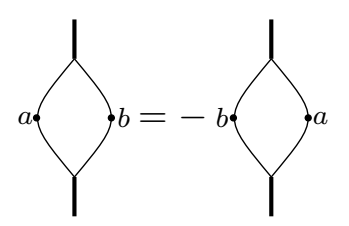

This antisymmetry implies the following:

\section{Lemma 1}

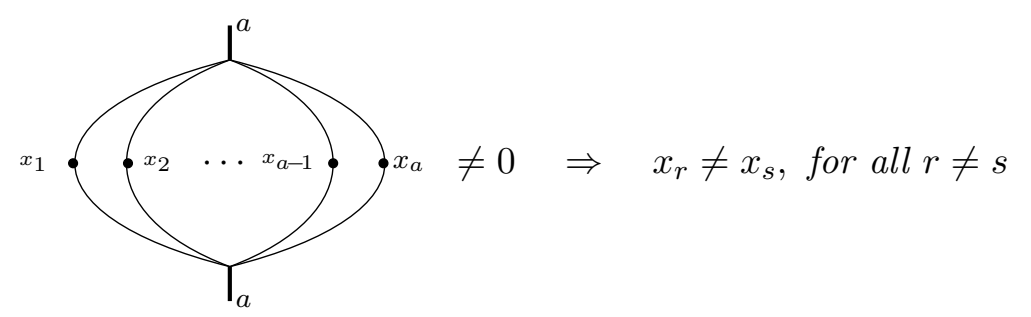

Moreover, if $\max _{i}\left\{x_{i}\right\}=a-1$, then the diagram from above can be nonzero if and only if there exists a permutation $\sigma$ of $\{0,1, \ldots, a-1\}$, such that $x_{a-i}=\sigma_{i}, i=0, \ldots, a-1$, in which case

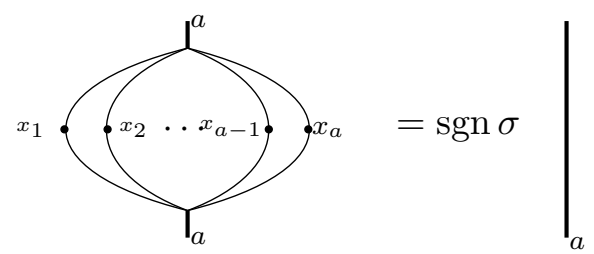

The above lemma implies the following

Lemma 2 [6, Proposition 2.4.1] Let $\alpha \in P(a, x)$ and $\beta \in P(b, y)$ be partitions. Then we have that

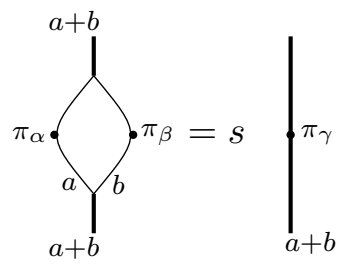


for some partition $\gamma \in P(a+b, \max \{x-b, y-a\})$ and $s \in\{-1,0,1\}$. If $s \neq 0$, then $|\gamma|=|\alpha|+|\beta|-a b$.

Moreover, if $\alpha \in P(a, b)$ and $\beta \in P(b, a)$, then we have:

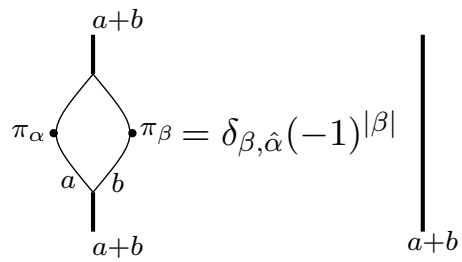

In particular, the left hand side can be nonzero only when $|\alpha|+|\beta|=a b$.

Lemma 3 [12] Let $\gamma \in P(a)$ and $\psi \in P(a, b)$ be partitions. Then

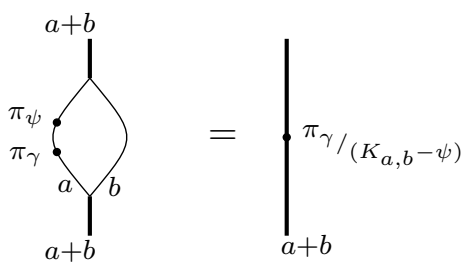

So far we have been examining the diagrams of a single color. We use the following convention when drawing the diagrams involving two adjacent colors.

Notation convention: For two colors (indices) that satisfy $i \cdot j=-1$, we shall draw strands colored $i$ as straight lines, and strands colored $j$ as curly lines:

$$
\left.\operatorname{Id}_{\mathcal{E}_{i}^{(a)}}:\left.\right|_{a} \quad \operatorname{Id}_{\mathcal{E}_{j}^{(b)}}:\right\}_{b}
$$

Thus, from now on, each line carries one label, and that label represents the thickness of a line.

The first "thick" property that we shall frequently use is about sliding the thick dots past crossings which involve strands of different colors. It follows straightforward from the analogous property for thin strands (8), the definition of thick dot (13) and associativity of splitters.

Proposition 5 (Dot Slide) The thick dots can be freely moved through the thick crossing of the two thick strands with different colors, i.e.: 


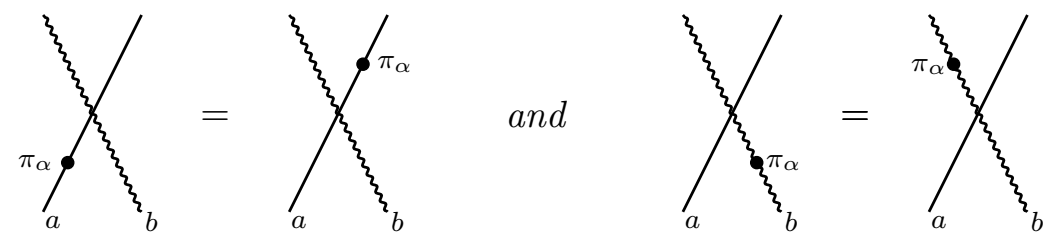

The following two propositions are extensions of the thin R2 and R3 relations (7) and (9).

Proposition 6 (Thick R2 Move) We have

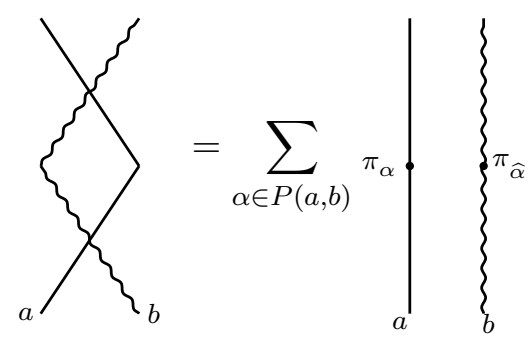

Proposition 7 (Thick R3 Move) We have

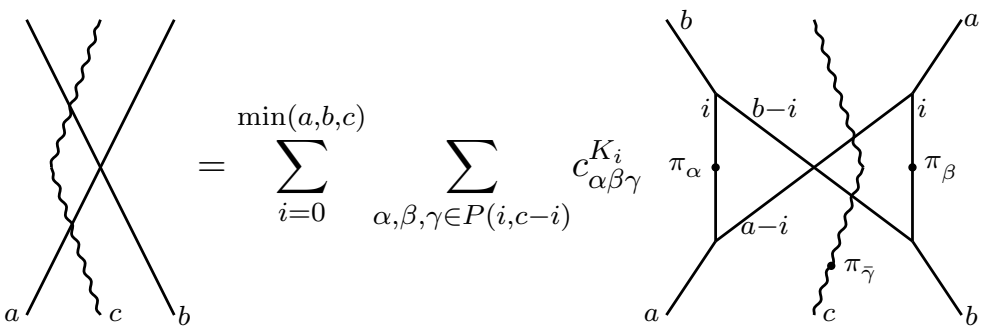

where $K_{i}=(\underbrace{c-i, c-i, \ldots, c-i}_{i})$, for $i>0$, and $K_{0}=0$.

\section{Proof of Proposition 6] - Thick R2 move}

First we prove the case $a=1$. We rewrite the left hand side by "exploding" the thick edge into $b$ thin strands by using (13) for $\alpha=0$, then we use the pitchfork lemma and dot slide, and then apply thin R2 move (77) $b$ times: 

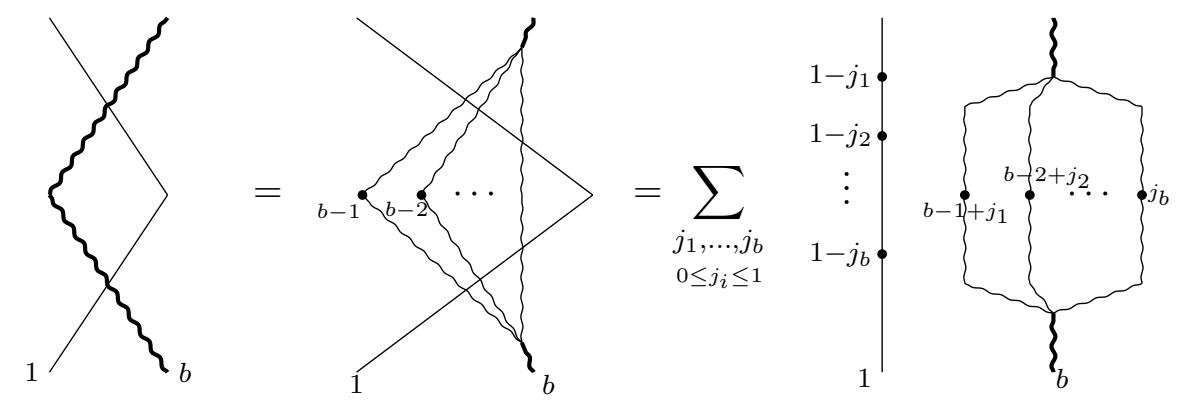

Since the "exploded" thick strand is antisymmetric with respect to the exchange of the number of dots on two thin strands, we have that the last diagram is zero whenever we have two strands with the same number of dots. Since the number of dots on $i$-th strand is equal to $b-i+j_{i}, i=1, \ldots, b$, and since all $j_{i}$ 's are either 0 or 1 , the last diagram can be nonzero if and only if $j_{1}=j_{2}=\ldots=j_{k}=1$ and $j_{i}=0$, for $i>k$, for some $k=0, \ldots, b$. Therefore, the last sum becomes simply

$$
\left.\sum_{l+k=b} l\right|_{1}\left\{\varepsilon_{b}\right.
$$

where $\varepsilon_{k}$ is the $k$-th elementary symmetric polynomial, as wanted.

As for the general case, now we split the strand of thickness $a$ into $a$ thin lines, and again after using pitchfork lemma and dot slides, we apply the thin R2 move $a$ times on the diagram involving strands of thicknesses 1 and $b$ :
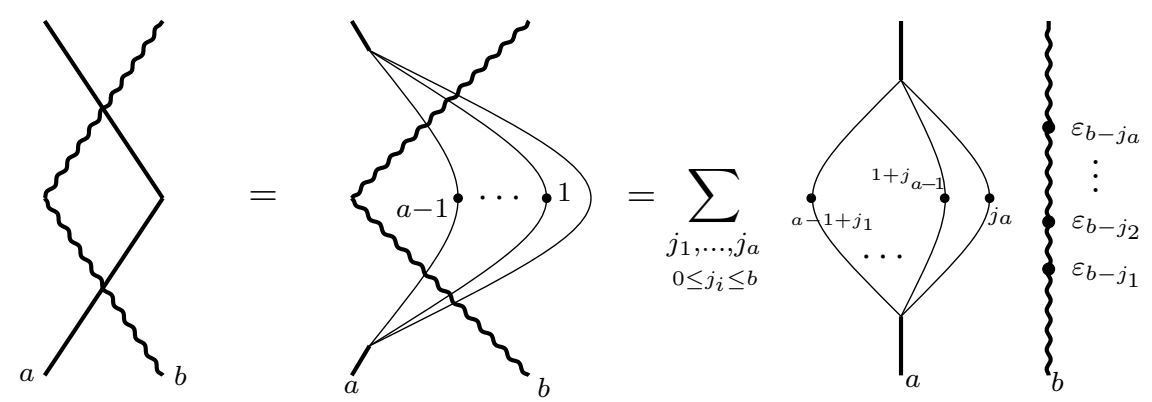

Since $\varepsilon_{k}$ as a polynomial in $b$ variables is zero whenever $k<0$ or $k>b$, in the last expression we can take summations over all integers $j_{i}$. Let $x_{i}=a-i+j_{i}, i=1, \ldots, a$. Then the last expression becomes 


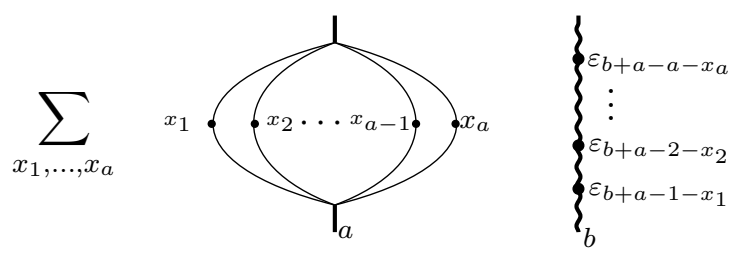

Due to antisymmetry of thin lines in the "exploded" thick strand, this can be nonzero only when all $x_{i}$ 's are pairwise distinct. Moreover, if we denote the decreasing ordering of such $x_{i}$ 's by $y_{1}>y_{2}>\ldots>y_{a}$, then for some permutation $\sigma \in S_{a}$, we have $x_{i}=y_{\sigma_{i}}, i=1, \ldots, a$, and the whole expression becomes

$$
\sum_{y_{1}>\cdots>y_{a}} \sum_{\sigma \in S_{a}} \operatorname{sgn} \sigma y_{1}\left\{y_{2} \ldots y_{a-1}, y_{a}\left\{\begin{array}{c}
\varepsilon_{b+a-a-y_{\sigma}} \\
\vdots \\
\varepsilon_{b+a-2-y_{\sigma_{2}}} \\
\varepsilon_{b+a-1-y_{1}} \\
b
\end{array}\right.\right.
$$

By setting $\alpha_{i}=y_{i}-(a-i), i=1, \ldots, a$, and $\alpha=\left(\alpha_{1}, \ldots, \alpha_{a}\right)$, by (13) we have

$$
\left.\sum_{\alpha \in P(a)} \sum_{\sigma \in S_{a}} \operatorname{sgn} \sigma \pi_{\alpha}\right|_{a}\left\{\begin{array}{c}
\varepsilon_{b-a-\alpha_{\sigma_{a}}+\sigma_{a}} \\
\vdots \\
\varepsilon_{b-2-\alpha_{\sigma_{2}}+\sigma_{2}} \\
\varepsilon_{b-1-\alpha_{\sigma_{1}}+\sigma_{1}}
\end{array}\right.
$$

Now, from the definition of the determinant of a matrix we have

$$
\begin{gathered}
\sum_{\sigma \in S_{a}} \operatorname{sgn} \sigma \prod_{i=1}^{a} \varepsilon_{b-i-\alpha_{\sigma_{i}}+\sigma_{i}}=\operatorname{det}\left[\varepsilon_{b-i-\alpha_{j}+j}\right]_{i, j=1}^{a}= \\
=\operatorname{det}\left[\varepsilon_{b-\alpha_{a+1-i}+j-i}\right]_{i, j=1}^{a}=\pi \frac{\left(b-\alpha_{a}, \ldots, b-\alpha_{2}, b-\alpha_{1}\right)}{} .
\end{gathered}
$$

In the second equality we used the fact that the determinants of a matrix and its transpose are equal, and that the determinant doesn't change if we invert the orders of the rows and of the columns of a matrix. Finally, the last equality is the Giambelli formula (12). Therefore, in the last diagram, a summand can be nonzero only for $\alpha \in P(a, b)$, and thus we get the desired equality. 


\section{Proof of Proposition 7 - Thick R3 move}

We will prove the proposition by induction on the thicknesses of the three strands involved. We shall frequently use the following

$$
\left.\right|_{a+1}=a \int_{a+1}^{a+1}
$$

which follows directly from (13) and Associativity of splitters.

First we prove the case when $a=c=1$, i.e. we show that for any $b$ :
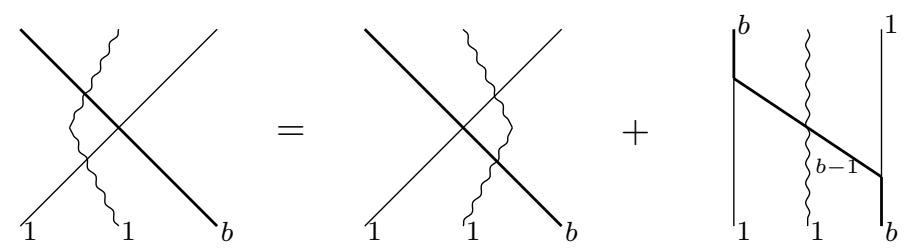

For $b=1$ this is simply the thin R3 relation (9). Now, suppose that (15) is valid for some $b \geq 1$, and we shall prove that it holds for $b+1$, as well. We start by using (14) on the strand of thickness $b+1$ :
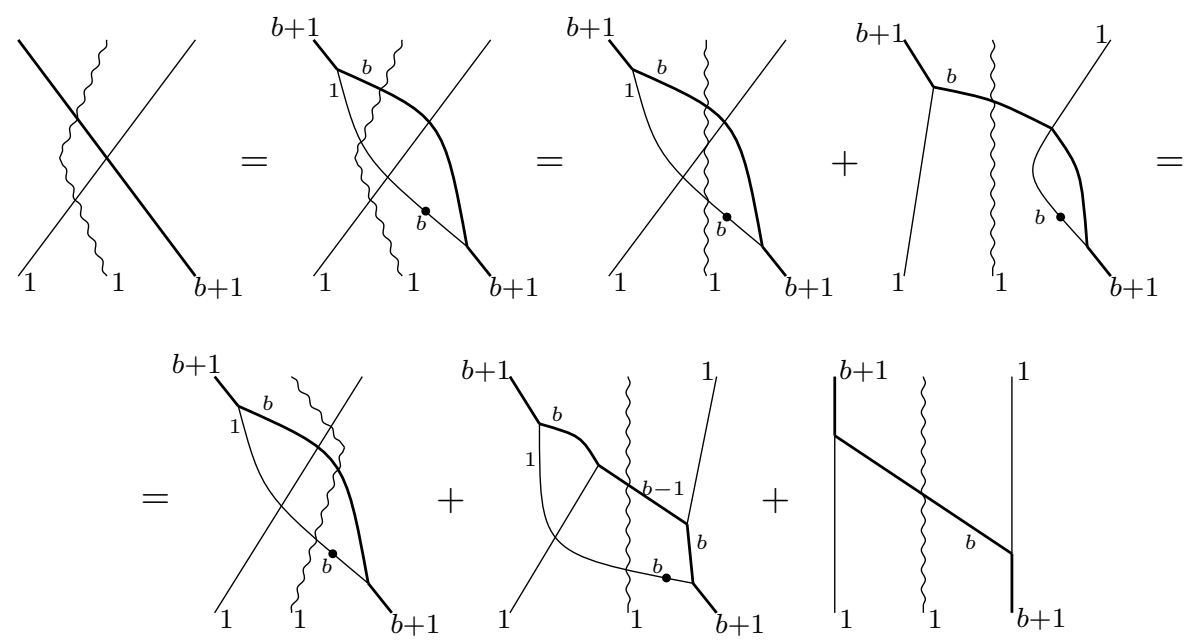

In the first equality we have used the pitchfork lemma, while the second one follows from the thin R3 move. The third equality follows by applying the induction hypothesis on the first diagram, and by using (14) on the second diagram. The first diagram is exactly the wanted first term on the right hand side of (15), by pitchfork lemma and (14). Finally, the middle diagram in the last expression after applying the associativity of splitters becomes 


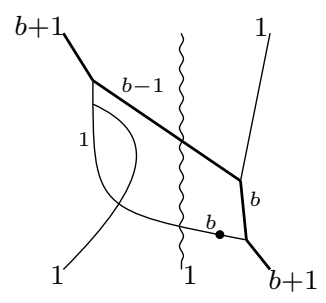

and is equal to zero since it contains a dotless digon (Lemma 2).

Now, we pass to the case $c=1$, i.e. we shall prove

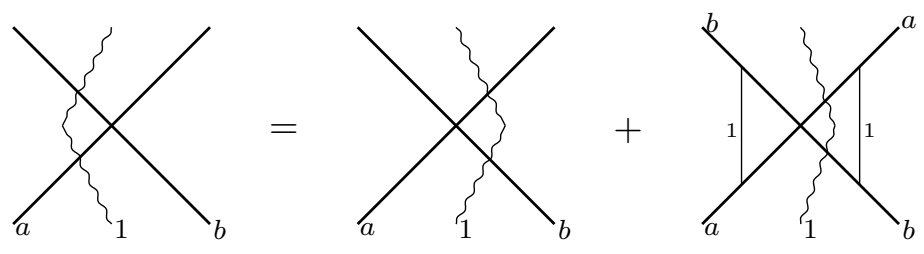

We prove this formula by induction on $a$. We assume that (16) is valid for some $a \geq 1$ and prove that it also holds for $a+1$. We rewrite the strand of thickness $a+1$ by using (14), and after performing dot slide and pitchfork lemma, we get

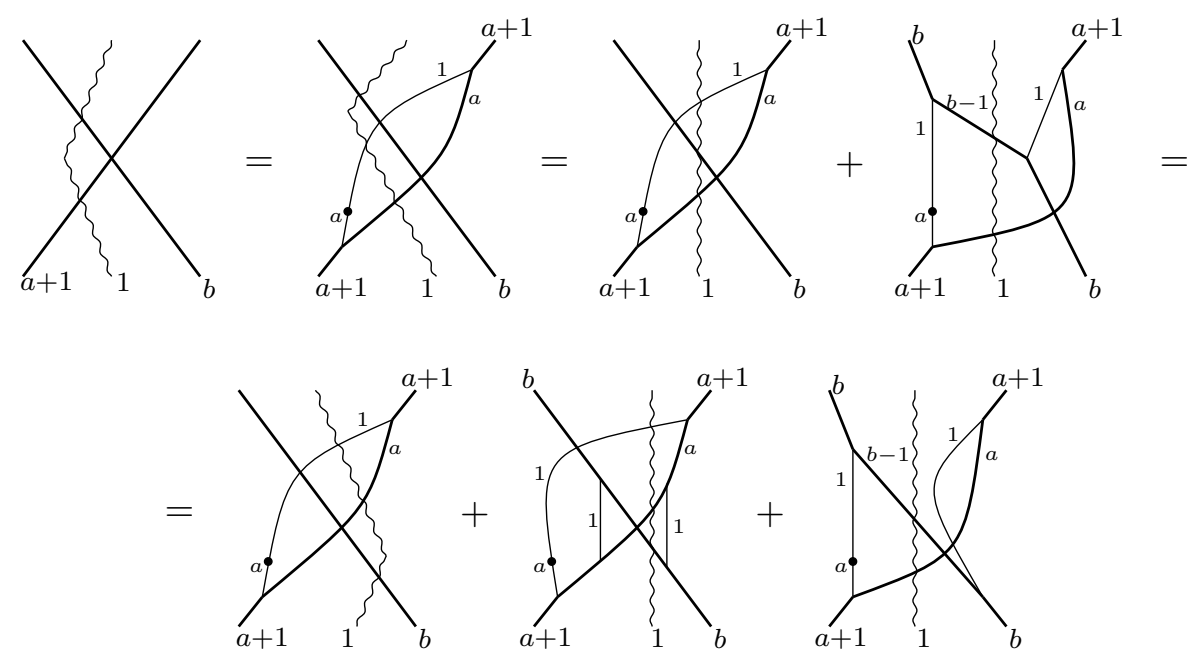

The second equality follows from (15). The third equality follows by applying the induction hypothesis on the first diagram, and by performing pitchfork lemma on the second diagram. The third of the three obtained summands is equal to zero since it contains a dotless digon, while the first summand equals the wanted first term from (16) by using pitchfork lemma and (14). The remaining (second) summand, after applying associativity of splitters and pitchfork lemma becomes: 

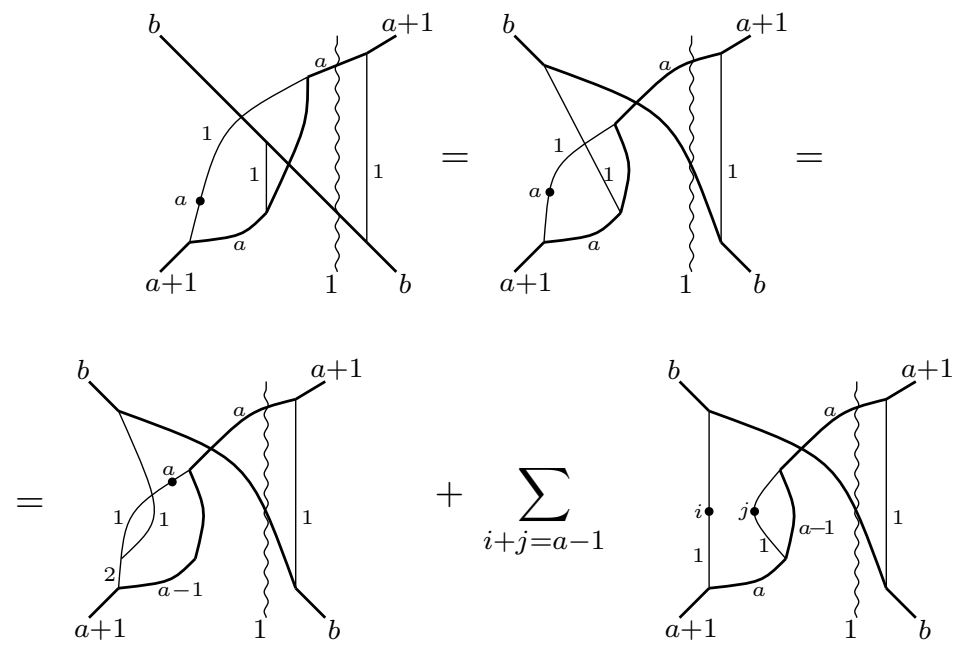

The first equality above follows by applying the pitchfork lemma twice, while the second one follows from Dot Migration. In the first diagram of the last line we have also used one associativity of splitters, and that diagram is equal to zero since it contains a dotless digon. Finally, all summands in the last summation with $j<a-1$ are zero by Lemma 2 due to the presence of a digon with the edges of thicknesses 1 and $a-1$, and so finally by (14) the last diagram is equal to the second summand on the RHS of (16), thus proving this case.

Finally, we are left with the general case. Now, we "explode" the curly strand of thickness $c$ into $c$ thin lines. We shall "pass" each of these $c$ thin lines through the thick crossing by using (16). There are two summands on the right-hand-side of (16) - the first one which is just simple passing of a thin curly strand, and the second extra-term. In all of such obtained summands for $c$ thin strands, either all of them have simply passed through a the thick crossing, or at least for some of the thin strands we have an extra term from (16). In the latter case, let the first strand that produces an extra term be the $j$-th one (counting from right to left). Then we have:
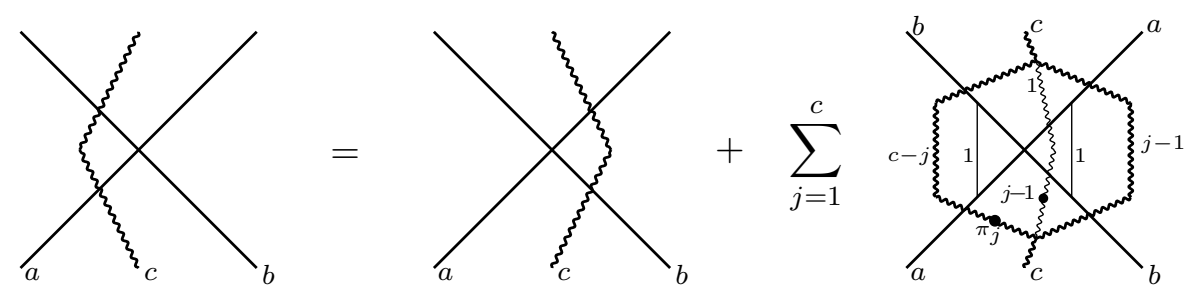

Here we have denoted $\pi_{j}:=\pi_{K_{c-j, j}}$ and we have also used the associativity of splitters and (13) to collect the different thin strands. Recall that $K_{p, q}$ denotes the partition $(q, q, \ldots, q)$ of length $p$. Now, we use the pitchfork 
lemma followed by the Thick R2 move on the curly strand of thickness $c-j$ and the left-most strand of thickness 1 , as well as on the curly strand of thickness $j-1$ and the right-most strand of thickness 1 .
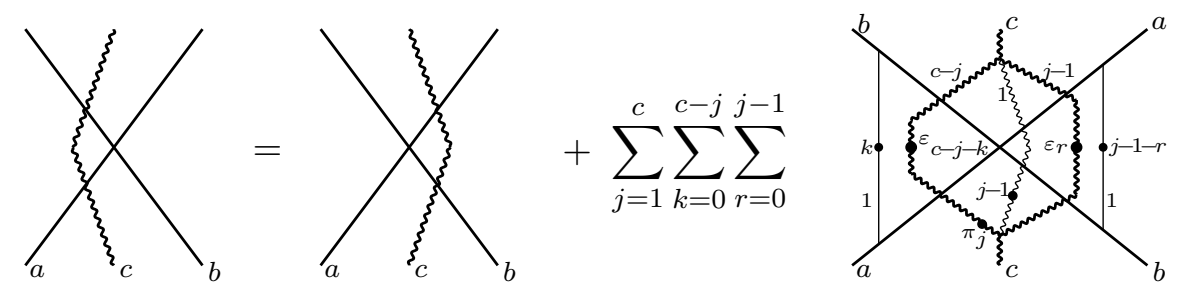

Now, as in the proof of the Thick R2 move, the last diagram can be nonzero only for $r=0$, since otherwise we would have two thin curly strands in the "exploded" thick one, both with $j-1$ dots and therefore equal to zero by antisymmetry. Therefore we have:
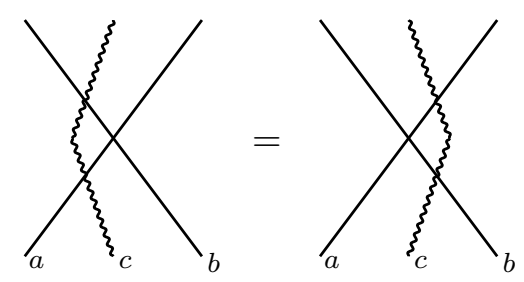

$$
+\sum_{j=1}^{c} \sum_{k=0}^{c-j}
$$

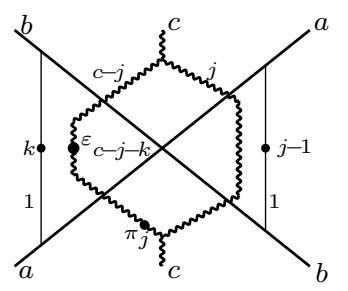

We can now iterate this formula, by passing the thick curly strand of thickness $c-j$ through the thick crossing by using analogous formula (17). In such a way we get (we are also applying dot slides):

$$
\sum_{a}=\sum_{x=0}^{c} \sum_{1 \leq j_{1}<\cdots<j_{x} \leq c} \sum_{k_{1}, \ldots, k_{x}}
$$




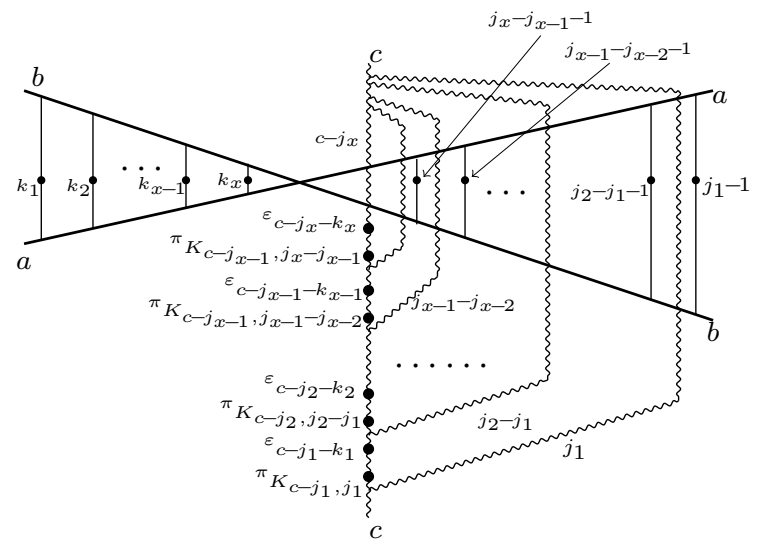

First of all, we note that we can assume that the summation is over all integers $k_{i}$, since the corresponding elementary symmetric polynomial $\varepsilon_{c-j_{i}-k_{i}}$ is equal to zero when $j_{i}+k_{i}$ is larger than $c$.

Now we apply the thick R2 move (after two pitchfork lemmas) on the curly strand of thickness $j_{1}$ and thin strand with $j_{2}-j_{1}-1$ dots.

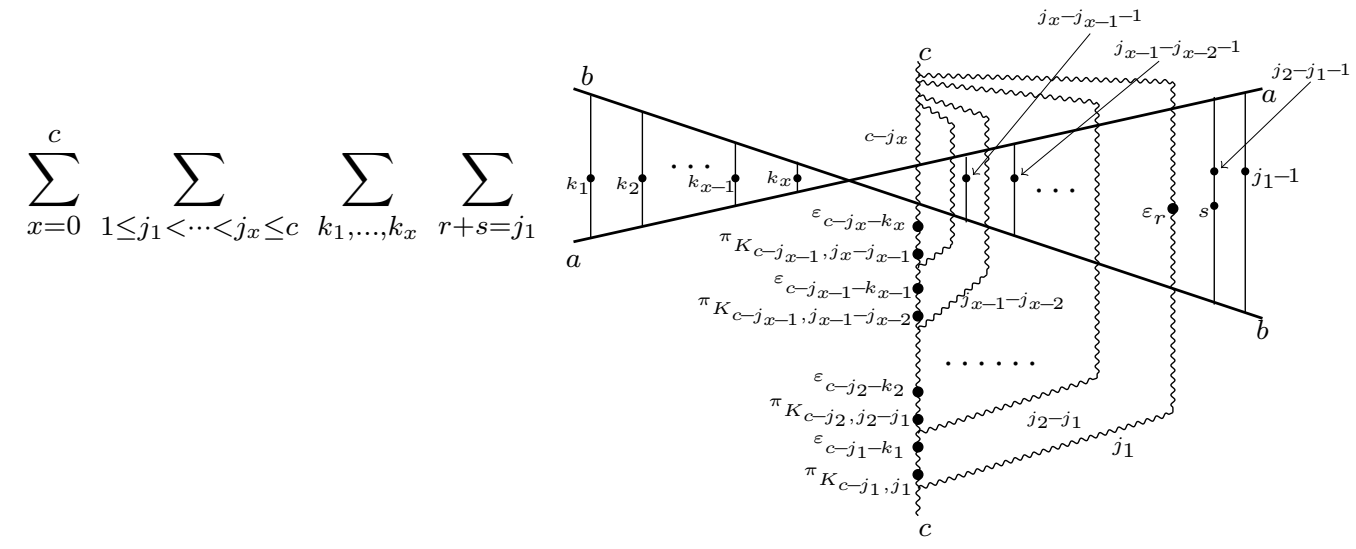

(19)

We will show that from all the summands appearing in this thick R2 move only the one with no dots on the thick curly line of thickness $j_{1}$ can be nonzero. Indeed, otherwise we move the thick dots through splitters by using [6, formulas (2.68) and (2.63)]:
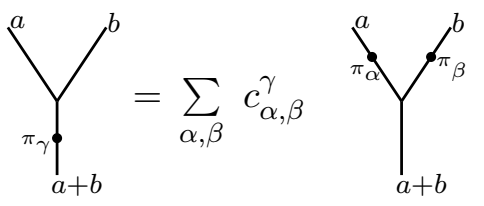

and

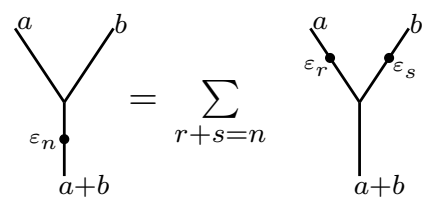


Then, a part of the diagram containing thick curly edges of thicknesses $j_{1}$ and $j_{2}-j_{1}$, after moving up the thick dots labelled by $\varepsilon_{c-j_{1}-k_{1}}$ and $\pi_{K_{c-j_{1}} j_{1}}$ and dot sliding of $\varepsilon_{r}$, by associativity of splitters becomes:

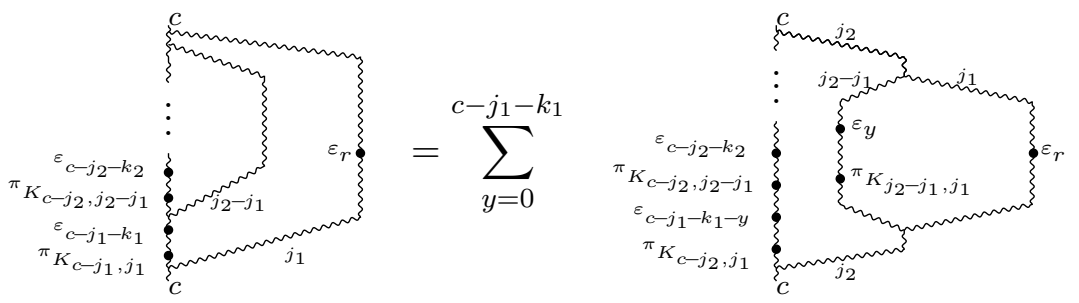

Again, for $r>0$ due to antisymmetry the bubble can be nonzero only if $y=j_{2}-j_{1}$. But then the expression on the curly strands is symmetric in $k_{1}$ and $k_{2}$ (the only dependence on them is the product $\varepsilon_{c-j_{2}-k_{1}} \varepsilon_{c-j_{2}-k_{2}}$ ), whereas it is antisymmetric on the ordinary strands on the left of the diagram in (19), and therefore the whole diagram is zero.

Hence, only the summand with $r=0$ survives in (19), and then by using Lemma 3 the bubble appearing in the above picture becomes just the thick strand of thickness $j_{2}$ with the dot labelled by $\varepsilon_{y}$. Finally, after moving down the dots decorated by $\varepsilon_{y}$ and $\varepsilon_{c-j_{1}-k_{1}-y}$ throughout the splitter, we get that (19) is equal to (note that $r=0$ and so $s=j_{1}$ )

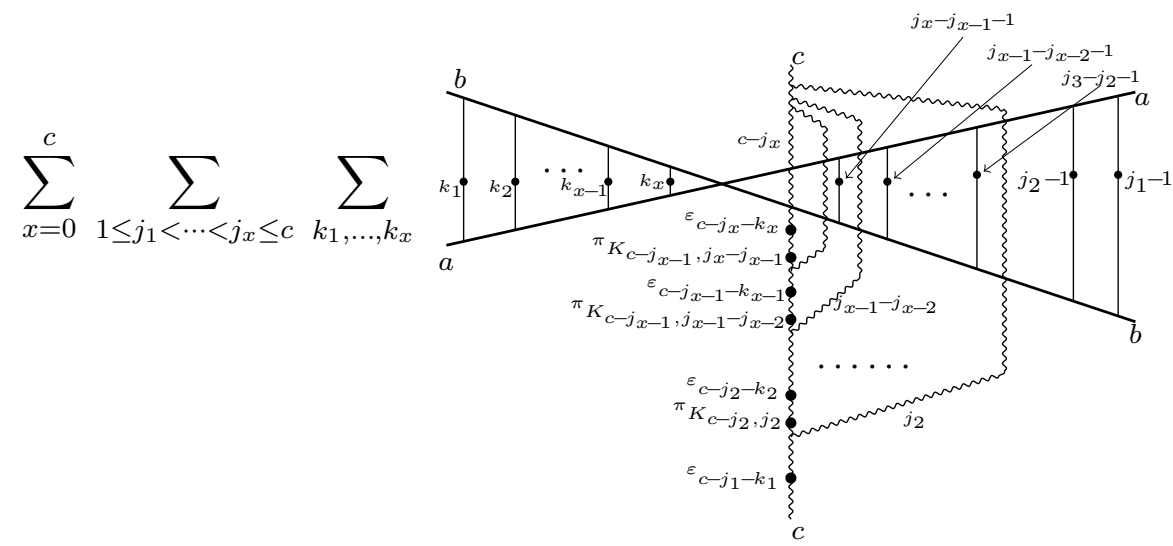

Now we can repeat the analogous thick R2 moves (preceded by the pitchfork lemma moves) in order to move all remaining thick curly strands to the left, starting with the strand of thickness $j_{2}$. In such a way we arrive at 


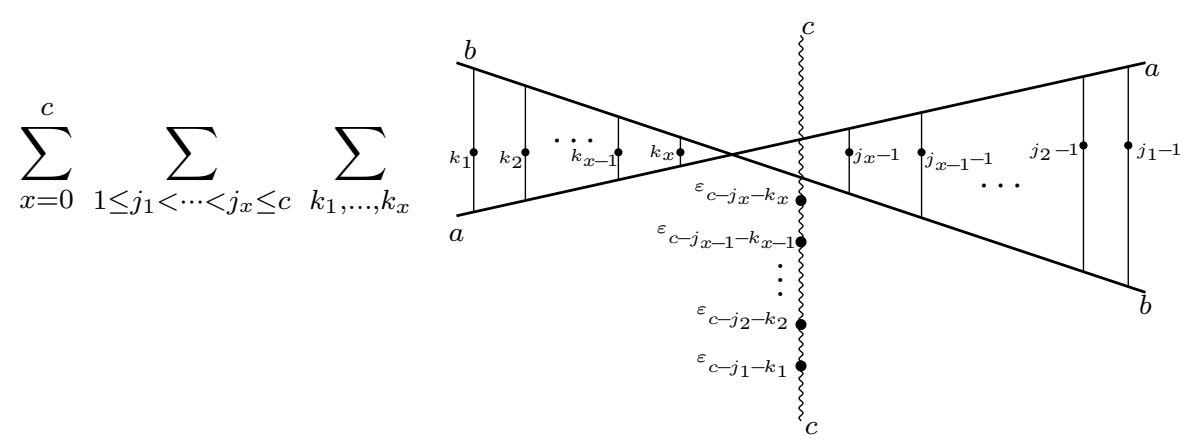

By using the associativity of splitters, we rewrite both groups of $x$ thin lines (the ones with labels $k_{i}$ and the other group with the labels $j_{i}-1$ ) as the exploded edges of thickness $x$. We do a similar thing as in the proof of the thick R2 move: first we note that due to the antisymmetry, the only nonzero summands are the ones where all $k_{i}$ are pairwise distinct, and we denote their decreasing ordering by $l_{1}>\ldots>l_{x}$. Let $\sigma \in S_{x}$ be a permutation such that $k_{i}=l_{\sigma_{i}}, i=1, \ldots, x$. Then let $\alpha=\left(\alpha_{1}, \ldots, \alpha_{x}\right)$ and $\beta=\left(\beta_{1}, \ldots, \beta_{x}\right)$ be partitions defined by $\alpha_{i}+x-i=l_{i}$ and $\beta_{x+1-i}=j_{i}-i$, $i=1, \ldots, x$, respectively. Then the last expression becomes simply:

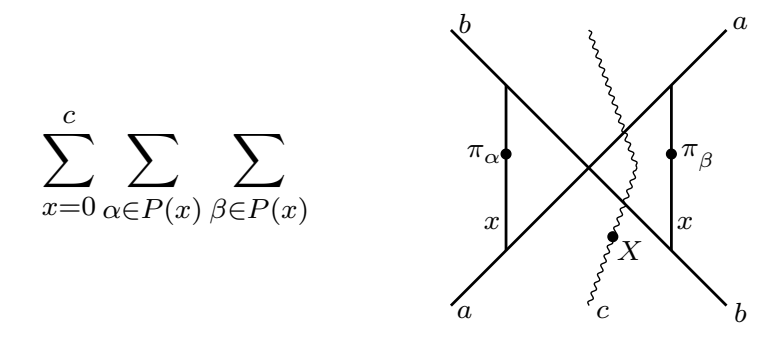

where

$$
X=\sum_{\sigma \in S_{x}} \operatorname{sgn}(\sigma) \prod_{i=1}^{x} \varepsilon_{c-\beta_{x+1-i}-i-\alpha_{\sigma_{i}}-x+\sigma_{i}} .
$$

Finally, we have

$$
\begin{aligned}
X= & \sum_{\sigma \in S_{x}} \operatorname{sgn}(\sigma) \prod_{i=1}^{x} \varepsilon_{c-\beta_{x+1-i}-i-\alpha_{\sigma_{i}}-x+\sigma_{i}}= \\
& =\operatorname{det}\left[\varepsilon_{c-\beta_{x+1-i}-i-\alpha_{j}-x+j}\right]_{i, j=1}^{x}= \\
& =\operatorname{det}\left[\varepsilon_{\left(c-x-\beta_{x+1-i}\right)-\alpha_{j}+j-i}\right]_{i, j=1}^{x}= \\
& =\operatorname{det}\left[\varepsilon_{\left(K_{x}-\beta\right)_{i}-\alpha_{j}+j-i}\right]_{i, j=1}^{x}=\pi_{\overline{K_{x}-\beta} / \bar{\alpha}} .
\end{aligned}
$$

The last equality follows from the expression of the skew Schur polynomial as a determinant of the elementary symmetric polynomials [10, formula (5.4), 
pp.70]. Finally, by definition, the last skew Schur polynomial can be written as follows:

$$
\begin{array}{r}
\pi_{\overline{K_{x}-\beta} / \bar{\alpha}}=\sum_{\gamma} c_{\bar{\alpha}, \bar{\gamma}}^{\overline{K_{x}-\beta}} \pi_{\bar{\gamma}}=\sum_{\gamma} c_{\alpha, \gamma}^{K_{x}-\beta} \pi_{\bar{\gamma}}= \\
=\sum_{\gamma} c_{\alpha, \gamma}^{K_{x}-\beta} c_{\beta, K_{x}-\beta}^{K_{x}} \pi_{\bar{\gamma}}=\sum_{\gamma} c_{\alpha, \beta, \gamma}^{K_{x}} \pi_{\bar{\gamma}},
\end{array}
$$

which finishes our proof.

\section{Additional thick relations}

The above thick R2 and R3 moves enable further relations for the general "passage" of a curly strand through a thick edge, i.e. moving a strand labeled $c$ to the other side of the thick edge labeled $t$ in the picture below:

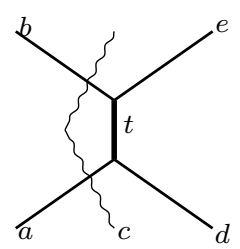

with $a+b=d+e=t$.

Depending on the sign of the difference $e-a=b-d$, we have two slightly different formulas for this moving. Both results are given in the following proposition.

Proposition 8 Let $a, b, c$ and $x$ be nonnegative integers. Then the following two relations hold in $\dot{\mathcal{U}}$ :
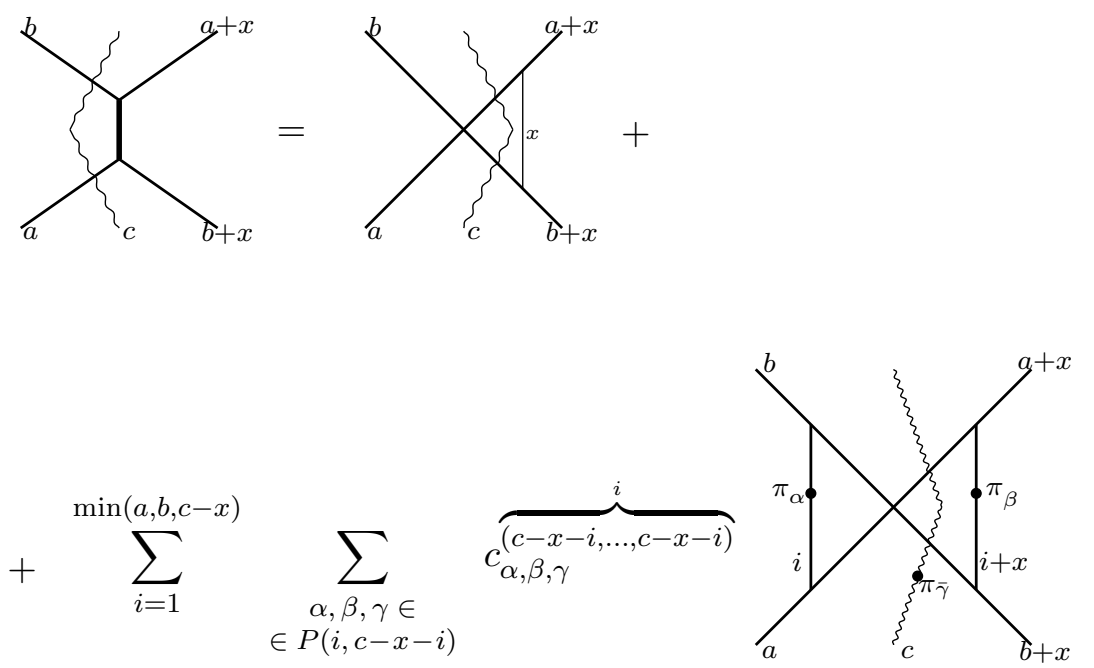


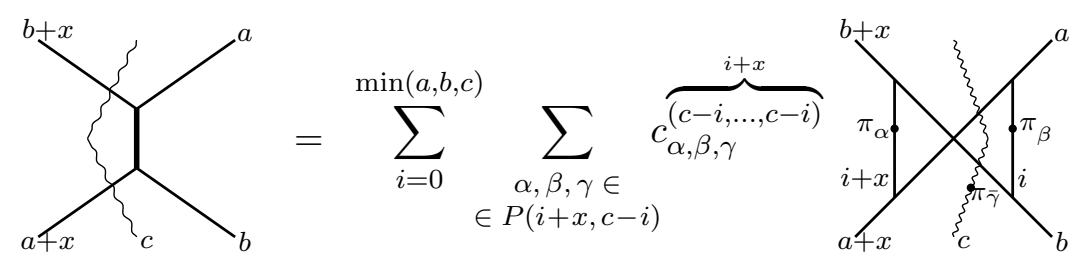

\section{Proof:}

First we prove the formula (201). We note that by (4):

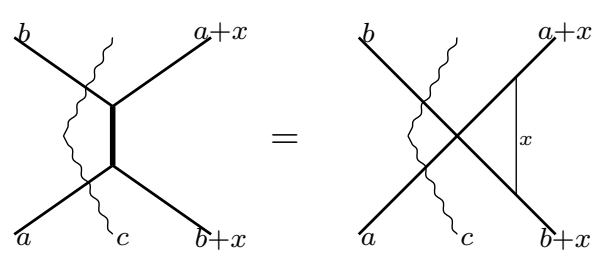

Now, by Thick R3 move (7) we get that the RHS of (22) equals:

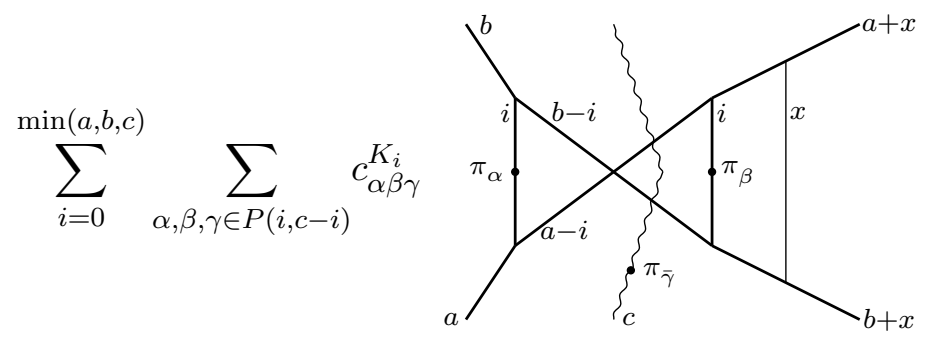

where $K_{i}=(\overbrace{c-i, c-i, \ldots, c-i}^{i})$, for $i>0$, and $K_{0}=0$. By Associativity of splitters (Proposition 2) and Lemma 3, the formula (23) simplifies to:

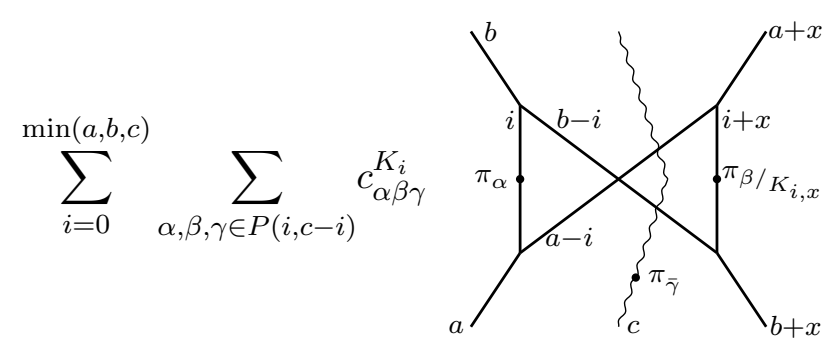

The last expression equals the RHS of (20), as wanted.

The formula (21) is proved analogously: again, by Proposition 4 we have: 


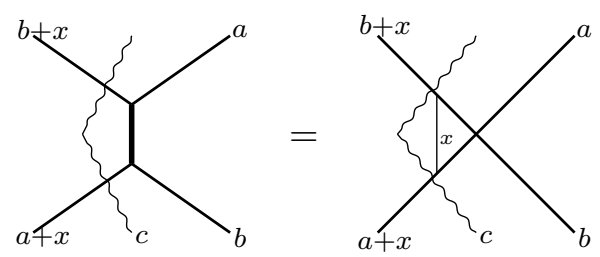

By Pitchfork Lemma and Thick R2 move, the RHS of (25) becomes:

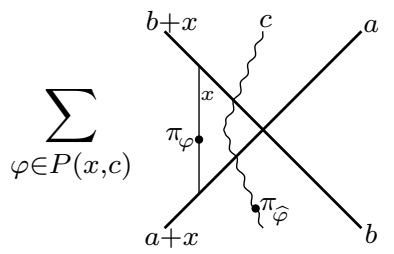

Recall, that by convention $\widehat{\varphi}=\overline{K_{x, c}-\varphi}$. Now, by Thick R3 move, the last expression is equal to

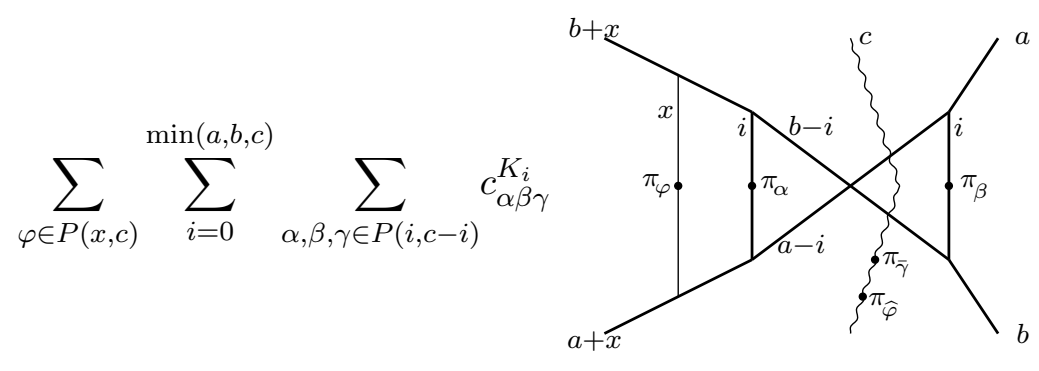

Again by applying the Associativity of Splitters, and grouping the summand analogously as before, we obtain (21).

\section{References}

[1] J. Brundan, A. Kleshchev: Graded decomposition numbers for cyclotomic Hecke algebras, Advances in Math. 222 (2009), 1883-1942.

[2] W. Fulton: Young Tableaux: with Applications to Representation Theory and Geometry, Cambridge University Press, Cambridge, 1997.

[3] M. Khovanov, A. Lauda: A diagrammatic approach to categorification of quantum groups I, Represent. Theory 13 (2009), 309-347.

[4] M. Khovanov, A. Lauda: A diagrammatic approach to categorification of quantum groups II, Trans. Amer. Math. Soc. 363 (2011), 2685-2700.

[5] M. Khovanov, A. Lauda: A diagrammatic approach to categorification of quantum groups III, Quantum Topology, Vol 1, Issue 1 (2010), 1-92. 
[6] M. Khovanov, A. Lauda, M. Mackaay, M. Stošić: Extended graphical calculus for categorified quantum sl(2), Memoirs of the AMS, Vol. 219, No. 1029 (2012).

[7] A. Lauda: A categorification of quantum sl(2), Advances in Mathematics, Volume 225, Issue 6 (2010), 3327-3424.

[8] G. Lusztig: Introduction to quantum groups, volume 110 of Progress in Mathematics, Birkhäuser, Boston, 1993.

[9] G. Lusztig: Canonical bases arising from quantized enveloping algebras, J. Amer. Math. Soc. 3 (1990), 447-498.

[10] I.G. Macdonald: Symmetric functions and Hall polynomials, Oxford Mathematical Monographs, Clarendon Press, Oxford, 1995.

[11] H. Queffelec, D. Rose: The $\mathfrak{s l}_{n}$ foam 2-category: a combinatorial formulation of Khovanov-Rozansky homology via categorical skew Howe duality, arXiv:1405.5920.

[12] M. Stošić: Indecomposable objects and Lusztig's canonical basis, Math. Res. Lett. 22, no. 1 (2015), 245-278.

[13] M. Varagnolo, E. Vasserot: Canonical basis and KLR-algebras, J. Reine Angew. Math. 659 (2011), 67-100.

[14] B. Webster: Knot invariants and higher representation theory, arXiv:1309.3796.

CAmgsd, Departamento de Matemática, instituto Superior Técnico, Av. Rovisco Pais, 1049-001 Lisbon, Portugal,

and

Mathematical Institute SAnU, Knez Mihailova 36, 11000 Beograd, Serbia.

E-mail: mstosic@math.ist.utl.pt 